\title{
FRECUENCIA DE LAS MUTACIONES MÁS COMUNES DEL GEN CFTR EN PACIENTES PERUANOS CON FIBROSIS QUÍSTICA MEDIANTE LA TÉCNICA ARMS-PCR
}

\author{
Ruth Aquino ${ }^{1,2, a, h}$, Ana Protzel ${ }^{3, b, c}$, Juan Rivera ${ }^{4, b, d}$, Hugo Abarca ${ }^{4, e}$, Milagros Dueñas $^{3, e}$, Cecilia Nestarez $^{5, f}$, \\ Nestor Purizaga ${ }^{1, g}$, Benoit Diringer ${ }^{2, i}$
}

\begin{abstract}
RESUMEN
Objetivos. Determinar la frecuencia de las diez mutaciones más comúnmente reportadas en América Latina del gen CFTR mediante Sistema de Mutación Refractario a la amplificación por PCR (ARMS-PCR) en los pacientes con fibrosis quística (FQ) de dos instituciones hospitalarias de referencia en el Perú durante el año 2014. Materiales y métodos. Se evaluó la frecuencia de las diez comúnmente reportadas más comúnmente reportadas del gen CFTR en los pacientes del Hospital Nacional Edgardo Rebagliati Martins y el Instituto Nacional de Salud del Niño, ambos ubicados en Lima, Perú. Se recogieron muestras de sangre de 36 pacientes con FQ y se utilizó la técnica de ARMS-PCR para determinar la presencia de tales mutaciones. Resultados. Se incluyó al 73,5\% de los pacientes con diagnóstico conocido de $\mathrm{FQ}$ en el país al momento en que se realizó el estudio. El diagnóstico por ARMS-PCR permitió identificar las mutaciones en $30,6 \%$ de los alelos de los pacientes con FQ, el 64,9\% de los alelos mutados no fue identificado. Las mutaciones encontradas fueron p.Phe508del $(22,2 \%)$, p.Gly542* $(6,9 \%)$ y p.Arg $1162^{*}(1,4 \%)$. Conclusiones. Existe una variabilidad significativa de las mutaciones presentes en nuestra población de estudio en comparación con lo reportado en otros países de Latinoamérica, tanto en la frecuencia como en el tipo. Es necesario realizar estudios que usen la tecnología de secuenciación completa del gen CFTR para identificar otras mutaciones presentes en nuestra población.
\end{abstract}

Palabras clave: Fibrosis quística; Mutación; Diagnóstico, Perú (fuente: DeCS BIREME).

\section{FREQUENCY OF THE MOST COMMON MUTATIONS OF THE CFTR GENE IN PERUVIAN PATIENTS WITH CYSTIC FIBROSIS USING THE ARMS-PCR TECHNIQUE}

\begin{abstract}
Objectives. To determine the frequency of the ten most common mutations of the CFTR gene reported in Latin America using amplification-refractory mutation system-polymerase chain reaction (ARMS-PCR) in patients with cystic fibrosis (CF) in two referral hospitals in Peru during the year 2014. Materials and Methods. The frequency of the ten most common mutations of the CFTR gene was assessed in patients of the Hospital Nacional Edgardo Rebagliati Martins and the Instituto Nacional de Salud del Niño, both located in Lima, Peru. Blood samples were collected from 36 patients with $\mathrm{CF}$, and the ARMS-PCR technique was used to determine the presence of these mutations. Results. The study group included $73.5 \%$ of patients with a known diagnosis of CF in the country when the study was carried out. ARMS-PCR allowed three of the mutations to be identified in a combined $30.6 \%$ of the alleles from patients with $\mathrm{CF}$, and $64.9 \%$ of the mutated alleles were not identified. The mutations found were p.Phe508del (22,2\%), p.Gly542* (6,9\%), and p.Arg1162* $(1,4 \%)$. Conclusions. There is significant variability in both the frequency and type of mutations present in our study population and in what has been reported in other Latin American countries. It is necessary to perform studies that use complete sequencing technology for the CFTR gene to identify other mutations present in our population.
\end{abstract}

Keywords: Cystic Fibrosis; Mutation; Diagnosis, Peru (source: MeSH NLM).

\footnotetext{
Universidad Nacional de Tumbes. Tumbes, Perú.

Empresa de Investigación y Capacitación en Biotecnología Inca’Biotec. Tumbes, Perú.

Hospital Nacional Edgardo Rebagliati Martins. Lima, Perú.

Instituto Nacional de Salud del Niño. Lima, Perú.

Asociación de Padres de Niños con Fibrosis Quística. Lima, Perú.

Biólogo, ${ }^{\mathrm{b}}$ pediatra, ${ }^{\mathrm{c}}$ genetista, ${ }^{\mathrm{d}}$ gastroenterólogo, ${ }^{\mathrm{e}}$ médico genetista, ${ }^{\mathrm{f}}$ obstetra, ${ }^{\mathrm{g}}$ patólogo clínico, ${ }^{\mathrm{h}}$ magister en Biotecnología Molecular, ${ }^{\mathrm{i}}$ magíster en Ciencias de la Vida y de la Tierra

Recibido: 08/09/2016 Aprobado: 08/02/2017 En línea: 23/03/2017
}

Citar como: Aquino R, Protzel A, Rivera J, Abarca H, Dueñas M, Nestarez C, et al. Frecuencia de las mutaciones más comunes del gen CFTR en pacientes peruanos con fibrosis quística mediante la técnica ARMS-PCR. Rev Peru Med Exp Salud Publica. 2017;34(1):62-9. doi: 10.17843/rpmesp.2017.341.2767 


\section{INTRODUCCIÓN}

La fibrosis quística $(F Q)$ es una enfermedad congénita ocasionada por un gen autosómico recesivo que afecta a 1 de cada 3500 nacidos vivos a nivel mundial y cuya prevalencia es mayor entre poblaciones caucásicas (1). Las mutaciones que inducen la FQ han sido asociadas a defectos de una proteína reguladora del transporte de cloro codificada por el gen CFTR (en inglés: Cystic fibrosis transmembrane conductance regulator) ${ }^{(2)}$.

Las mutaciones en el CFTR causan un defecto en el transporte de agua dentro y fuera de las células, lo que resulta en exceso de secreción de moco anormalmente espeso y viscoso a nivel pulmonar, pancreático, e intestinal, y conlleva a complicaciones graves e incluso la muerte (1). A la fecha, más de 1900 mutaciones diferentes han sido identificadas en el gen CFTR, sin embargo, la deleción del codón para la fenilalanina en la posición 508 (p.Phe508del) es la más frecuente ${ }^{(1)}$.

Los orígenes de las mutaciones del CFTR son específicos a ciertas zonas geográficas; algunas pueden ser restringidas a ciertos países, regiones e incluso familias ${ }^{(3)}$. Las mutaciones encontradas entre los diferentes países de Latinoamérica reflejan la gran heterogeneidad genética de sus habitantes (4) y son probablemente heredadas de los flujos migratorios que ocurrieron en los últimos siglos ${ }^{(5)}$. La población peruana es el producto del mestizaje entre amerindios, españoles, africanos y asiáticos; por lo que se espera que existan variaciones importantes dentro del país y de una región a otra en relación al componente étnico que predomine en cada zona.

El tipo de mutaciones presente en ambos alelos del gen CFTR influye en la severidad del disfuncionamiento del canal de transporte del CFTR. Dicha variabilidad conduce a un cuadro clínico de expresión variable, por lo que la tipificación del perfil genético de cada paciente es fundamental para elegir las opciones terapéuticas, establecer el pronóstico y brindar la asesoría genética.

En Latinoamérica, el subdiagnóstico de FQ ocurre a causa de la falta de sospecha clínica y/o elementos adecuados para el diagnóstico ${ }^{(6)}$. En el Perú, la FQ es una enfermedad infradiagnosticada y con limitaciones de acceso a exámenes de diagnóstico como la prueba de sudor y estudios moleculares; así como el tamizaje neonatal con la prueba de tripsina inmunoreactiva (IRT) que se realiza sistemáticamente en países del hemisferio norte ${ }^{(7)}$. Además, no existen estudios de prevalencia ni de caracterización genética de la enfermedad. No obstante, según los datos estadísticos existentes para América Latina, en el Perú estarían naciendo de 60 a 120 niños con $\mathrm{FQ}$ cada año ${ }^{(7)}$.
Actualmente, en los países desarrollados, los individuos diagnosticados con $\mathrm{FQ}$ son sometidos a tratamientos terapéuticos avanzados que les permiten tener una esperanza de vida de 39 años ${ }^{(8)}$. En el Perú se conoce menos de 50 pacientes vivos con diagnóstico confirmado, y la gran mayoría son pacientes en edad pediátrica ${ }^{(9)}$.

Este estudio tiene por objetivo evaluar la frecuencia de diez de las mutaciones más comunes en América Latina ${ }^{(7)}$, mediante el Sistema de Mutación Refractario a la Amplificación por PCR (ARMS-PCR, por sus siglas en inglés) en los pacientes con $F Q$ en el Perú. Los resultados obtenidos en esta investigación son los primeros publicados en el país considerando un número significativo de pacientes.

\section{MATERIALES Y MÉTODOS}

\section{DISEÑO Y POBLACIÓN DE ESTUDIO}

Se realizó un estudio observacional transversal de tipo descriptivo. La población estuvo compuesta por pacientes con $\mathrm{FQ}$ registrados en los dos hospitales especializados de Lima que atienden a la mayoría de pacientes con $F Q$, el Hospital Nacional Edgardo Rebagliati Martins (HNERM), perteneciente al Seguro Social del Perú y el Instituto Nacional de Salud del Niño (INSN), del Ministerio de Salud; invitados a través de la Asociación Peruana de FQ (FIQUI-Perú). Los pacientes habían sido previamente diagnosticados mediante la prueba de sudor y no contaban con diagnóstico molecular. El estudio se realizó durante el año 2014. El muestreo fue por conveniencia debido a la baja prevalencia de la enfermedad.

\section{CRITERIOS DE SELECCIÓN}

Los criterios de inclusión fueron: haber sido atendido en las dos instituciones previamente detalladas (HNERM y INSN) durante el año 2014 y tener el diagnóstico de FQ con dos pruebas positivas de sudor de sudor ( $>$ $60 \mathrm{mEq} / \mathrm{L}$ ). Se excluyeron aquellos pacientes que no desearon participar en el estudio o se negaban a firmar el consentimiento informado.

\section{ANÁLISIS MOLECULAR}

Se recogió una muestra de sangre periférica de cada paciente; posteriormente, las muestras fueron enviadas al Laboratorio de Biotecnología molecular de la Empresa de Investigación y Capacitación en Biotecnología Inca'Biotec/Universidad Nacional de Tumbes. A cada muestra se les realizó una extracción de ADN de las células blancas de la sangre, de acuerdo a protocolos ya probados ${ }^{(10)}$. La calidad y concentración del ADN 
obtenido fue evaluada mediante espectrofotometría de luz ultravioleta con el biofotómetro (Eppendorf®).

El ADN aislado de cada paciente fue amplificado con la técnica de Reacción en cadena polimerasa de sistema de mutación refractario a la amplificación (ARMS-PCR) para identificar las diez mutaciones más comunes: p.Phe508del, c.489+1G>T, p.Gly551Asp, p.Asn1303Lys, p.Gly542*, p.Lys1177Serfs*15, c.15851G>A, p.Trp1282*, p.Arg553*, p.Arg1162*, usando el kit de ADN Platinum ${ }^{\circledR}$ de Invitrogen, a partir de protocolos modificados de las referencias ${ }^{(11,12)}$.

Para las mutaciones p.Phe508del, c.489+1G $>\mathrm{T}$, p.Asn1303Lys, p.Gly551Asp y p.Gly542*, se desarrollaron cuatro mezclas. En cada una de las mezclas se incluyeron $86 \mu \mathrm{M}$ de dNTPs, 1,5 mM de $\mathrm{MgCl}_{2}, 1 \mathrm{X}$ de buffer para PCR y $1 \mathrm{U} / \mu \mathrm{L}$ de taq ADN polimerasa. Adicionalmente, en la primera mezcla se agregaron cebadores para: p.Phe508del (normal: 1,5 $\mu \mathrm{M}$; común: 0,5 $\mu \mathrm{M}$ ), c.489+1G>T (normal: 0,5 $\mu \mathrm{M}$, común: 0,5), p.Asn1303Lys (normal: $2 \mu \mathrm{M}$; común: $2 \mu \mathrm{M})$ y $100 \mathrm{ng} / \mu \mathrm{L}$ de $\mathrm{ADN}$; en la segunda mezcla cebadores para: p.Phe508del (mutado: $2 \mu \mathrm{M}$; común: $2 \mu \mathrm{M}$ ), c.489+1G>T (mutado: $0,25 \mu \mathrm{M}$; común: 0,25 $\mu \mathrm{M}$ ), p.Asn1303Lys (mutado: $1 \mu \mathrm{M}$; común: $1 \mu \mathrm{M}$ ) y $100 \mathrm{ng} / \mu \mathrm{L}$ de $\mathrm{ADN}$; en la tercera mezcla cebadores para: p.Gly551Asp (mutado: 0,5 $\mu \mathrm{M}$; común: 0,5 $\mu \mathrm{M}$ ), p.Gly542* (mutado: 0,5 $\mu \mathrm{M}$ ) y $100 \mathrm{ng} / \mu \mathrm{L}$ de ADN; y en la cuarta mezcla cebadores para: G551D (normal: 0,25 $\mu \mathrm{M}$; común: 0,5 $\mu \mathrm{M}$ ), G542X (normal: $0,25 \mu \mathrm{M}$ ) y 100 $\mathrm{ng} / \mu \mathrm{L}$ de $\mathrm{ADN}$. Las cuatro mezclas tuvieron un volumen final de $20 \mu \mathrm{L}$ cada una. La amplificación se realizó en un termociclador (Biometra - USA), las condiciones térmicas fueron desnaturalización inicial a $94{ }^{\circ} \mathrm{C}$ durante 2 min (un ciclo), seguido de 35 ciclos de amplificación, desnaturalización a $94{ }^{\circ} \mathrm{C}$ durante $30 \mathrm{~s}$, hibridación a $61^{\circ} \mathrm{C}$ durante $30 \mathrm{~s}$, y extensión a $72{ }^{\circ} \mathrm{C}$ durante $30 \mathrm{~s}$, con una etapa de extensión final a $72{ }^{\circ} \mathrm{C}$ durante $10 \mathrm{~min}$.

Para las mutaciones p.Lys1177Serfs*15, c.1585-1G>A se realizaron dos mezclas. Ambas incluyeron $200 \mu \mathrm{M}$ de dNTPs, 1,5 mM de $\mathrm{MgCl}_{2}$, $1 \mathrm{X}$ de buffer para PCR y $1 \mathrm{U} /$ $\mu \mathrm{L}$ de taq $A D N$ polimerasa Platinum $\AA$. Adicionalmente, en la primera mezcla se incluyeron cebadores para: p.Lys1177Serfs*15 (normal: $2 \mu \mathrm{M}$; común: $2 \mu \mathrm{M}$ ), c.1585-1G>A (mutado: $1,5 \mu \mathrm{M}$; común: $1,5 \mu \mathrm{M}$ ) y 100 $\mathrm{ng} / \mu \mathrm{L}$ de $\mathrm{ADN}$; en la segunda mezcla cebadores para: p.Lys1177Serfs ${ }^{*} 15$ (mutado: 1,5 $\mu \mathrm{M}$ de; común: $1,5 \mu \mathrm{M}$ ), c. $1585-1 \mathrm{G}>\mathrm{A}$ (normal: 0,5 $\mu \mathrm{M}$; común: $1,5 \mu \mathrm{M}$ ) y $100 \mathrm{ng} /$ $\mu \mathrm{L}$ de ADN. El volumen final de cada una fue $20 \mu \mathrm{L}$. Las condiciones térmicas fueron desnaturalización inicial a $94^{\circ} \mathrm{C}$ durante 2 min (un ciclo), seguido de 35 ciclos de amplificación, desnaturalización a $94{ }^{\circ} \mathrm{C}$ durante $30 \mathrm{~s}$, hibridación a $65{ }^{\circ} \mathrm{C}$ durante $30 \mathrm{~s}$, y extensión a $72^{\circ} \mathrm{C}$ durante $30 \mathrm{~s}$, con una etapa de extensión final a $72{ }^{\circ} \mathrm{C}$ durante $10 \mathrm{~min}$.

Para el diagnóstico de la mutación p.Arg1162*, se prepararon dos mezclas. Ambas contenían $200 \mu \mathrm{M}$ de dNTPs, 3 mM de $\mathrm{MgCl}_{2}$, 1X de buffer para PCR, 1,25 U/ $\mu \mathrm{L}$ de taq $\mathrm{ADN}$ polimerasa Platinum $\AA$. Adicionalmente, en la primera mezcla se incluyeron cebadores para: p.Arg1162* (común: $10 \mu \mathrm{M}$; normal: $10 \mu \mathrm{M}$ ) y $100 \mathrm{ng} /$ $\mu \mathrm{L}$ de $\mathrm{ADN}$; en la segunda mezcla cebadores para: p.Arg1162* (común: $10 \mu \mathrm{M}$; mutado: $10 \mu \mathrm{M}$ ) y $100 \mathrm{ng} / \mu \mathrm{L}$ de ADN. El volumen final de cada una fue de $20 \mu \mathrm{L}$. Las condiciones térmicas fueron desnaturalización inicial a $94{ }^{\circ} \mathrm{C}$ durante 5 min (un ciclo), seguido de 35 ciclos de amplificación, desnaturalización a $95{ }^{\circ} \mathrm{C}$ durante $1 \mathrm{~min}$, hibridación durante $30 \mathrm{~s}$ (a $63^{\circ} \mathrm{C}$ para la primera mezcla y a $60{ }^{\circ} \mathrm{C}$ para la segunda mezcla), y extensión a $72{ }^{\circ} \mathrm{C}$ durante $30 \mathrm{~s}$, con una etapa de extensión final a $72{ }^{\circ} \mathrm{C}$ durante $10 \mathrm{~min}$.

Para la mutación p.Arg553*, se realizó el protocolo de Single ARMS-PCR en dos mezclas. Ambas contenían 86 $\mu \mathrm{M}$ de dNTPs, 1,5 mM de $\mathrm{MgCl}_{2}$, $1 \mathrm{X}$ de buffer de PCR, $1 \mathrm{U} / \mu \mathrm{L}$ de taq $\mathrm{ADN}$ polimerasa Platinum ${ }^{\circledR}$. Además, en la primera mezcla se agregaron cebadores para: p.Arg553* (común: 0,86 $\mu \mathrm{M}$; normal: 0,86 $\mu \mathrm{M}$ ), AAT1 $(0,86 \mu \mathrm{M})$, AAT2 $(0,86 \mu \mathrm{M})$ y $100 \mathrm{ng} / \mu \mathrm{L}$ de ADN; en la segunda mezcla cebadores para: p.Arg553* (común: $0,86 \mu \mathrm{M}$; mutado: 0,86 $\mu \mathrm{M})$, AAT1 $(0,86 \mu \mathrm{M})$, AAT2 $(0,86$ $\mu \mathrm{M})$ y $100 \mathrm{ng} / \mu \mathrm{L}$ de ADN. El volumen final de cada una fue de $20 \mu \mathrm{L}$. Las condiciones térmicas de amplificación para ambas mezclas fueron desnaturalización inicial a $95{ }^{\circ} \mathrm{C}$ durante 5 min (un ciclo), seguido de 35 ciclos de amplificación, desnaturalización a $94{ }^{\circ} \mathrm{C}$ durante $2 \mathrm{~min}$, hibridación a $60{ }^{\circ} \mathrm{C}$ durante $2 \mathrm{~min}$, y extensión a $72{ }^{\circ} \mathrm{C}$ durante $2 \mathrm{~min}$, con una etapa de extensión final a $72{ }^{\circ} \mathrm{C}$ durante $10 \mathrm{~min}$.

También se desarrolló el protocolo de Single ARMSPCR para la mutación p.Trp1282*, este se realizó en dos mezclas. Ambas contenían $86 \mu \mathrm{M}$ de dNTPs, 1,5 $\mathrm{mM}$ de $\mathrm{MgCl}_{2}, 1 \mathrm{X}$ de buffer para PCR, $1 \mathrm{U} / \mu \mathrm{L}$ de taq ADN polimerasa Platinum $®$. En la primera mezcla se incluyeron cebadores para: p.Trp1282* (común: 0,86 $\mu \mathrm{M}$; normal: 0,86 $\mu \mathrm{M})$, AAT3 $(0,86 \mu \mathrm{M})$, AAT4 (0.86 $\mu \mathrm{M})$ y $100 \mathrm{ng} / \mu \mathrm{L}$ de $\mathrm{ADN}$; la segunda muestra cebadores para: p.Trp1282* (común: 0,86; mutado: 0,86 $\mu \mathrm{M}$ ), AAT3 $(0,86 \mu \mathrm{M})$, AAT4 $(0,86 \mu \mathrm{M})$ y $100 \mathrm{ng} / \mu \mathrm{L}$ de ADN. Ambas mezclas se llevaron a cabo en un volumen final de $20 \mu \mathrm{L}$. Las condiciones térmicas de amplificación para ambas mezclas fueron desnaturalización inicial a $95{ }^{\circ} \mathrm{C}$ durante 5 min (un ciclo), seguido de 35 ciclos de amplificación, desnaturalización a $94{ }^{\circ} \mathrm{C}$ durante $45 \mathrm{~s}$, hibridación a $60{ }^{\circ} \mathrm{C}$ durante $45 \mathrm{~s}$, y extensión a $72{ }^{\circ} \mathrm{C}$ durante $45 \mathrm{~s}$, con una etapa de extensión final a $72{ }^{\circ} \mathrm{C}$ durante $10 \mathrm{~min}$. Las secuencias de los cebadores utilizados se presentan en la Tabla 1. 
Tabla 1. Secuencias de cebadores usados para la detección de mutaciones mediante la técnica ARMS-PCR.

\begin{tabular}{|c|c|c|}
\hline Mutación & & Secuencias de cebadores $\left(5^{\prime} \rightarrow 3^{\prime}\right)$ \\
\hline & Normal & GTA TCT ATA TTC ATC ATA GGAAAC ACC AC \\
\hline \multirow[t]{3}{*}{ p.Phe508del } & Común & GAC TTC ACT TCT AAT GAT GAT TAT GGG AG \\
\hline & Mutado & GTA TCT ATA TTC ATC ATA GGAAAC ACC AT \\
\hline & Normal & GTC TTT CTC TGC AAA CTT GGA GAT GTG C \\
\hline \multirow[t]{3}{*}{ c. $1585-1 \mathrm{G}>\mathrm{A}$} & Común & TAAAAT TTC AGC AAT GTT GTT TTT GAC C \\
\hline & Mutado & GTC TTT CTC TGC AAA CTT GGA GAT GTG T \\
\hline & Normal & CCC ATC ACT TTT ACC TTA TAG GTG GGC CT \\
\hline \multirow[t]{3}{*}{ p.Trp1282* } & Común & СCT GTG GTA TCA CTC CAAAGG CTT TCC AC \\
\hline & Mutado & CCT GTG GTA TCA CTC CAAAGG CTT TCC AT \\
\hline & Normal & CAT GCC AAC AGA AGG TAA ACC TTC CA \\
\hline \multirow[t]{3}{*}{ p.Lys 1177 Serfs*15 } & Común & TCT GCT AAC ACA TTG CTT CAG GCT \\
\hline & Mutado & CAT GCC AAC AGA AGG TAA ACC TTC A \\
\hline & Normal & TGC CAT GGG GCC TGT GCA AGG AAG TAT T \\
\hline \multirow[t]{3}{*}{$c .489+1 G>T$} & Común & TCA CAT ATG GTA TGA CCC TCT ATA TAA AC \\
\hline & Mutado & TGC CAT GGG GCC TGT GCA AGG AAG TAT T \\
\hline & Normal & ACT CAG TGT GAT TCC ACC TTC TAC \\
\hline \multirow[t]{3}{*}{ p.Gly542* } & Común & TAAAAT TTC AGC AAT GTT GTT TTT GAC C \\
\hline & Mutado & CAC TCA GTG TGA TTC CAC CTT CTC A \\
\hline & Normal & TTA TTT CAG ATG CGA TCT GTG AGC C \\
\hline \multirow[t]{3}{*}{ p.Arg1162* } & Común & AAT CAT AAC TTT CGA GAG TTG GCC \\
\hline & Mutado & TTA TTT CAG ATG CGA TCT GTG AGC TT \\
\hline & Normal & GCT AAA GAA ATT CTT GCT CGT TAC C \\
\hline \multirow[t]{3}{*}{ p.Gly551Asp } & Común & TAAAAT TTC AGC AAT GTT GTT TTT GAC C \\
\hline & Mutado & AGC TAAAGAAAT TCT TGC TCG TTG CT \\
\hline & Normal & CAC CTT GCT AAA GAAATT CTT GCT AG \\
\hline \multirow[t]{3}{*}{ p.Arg553* } & Común & TAAAAT TTC AGC AAT GTT GTT TTT GAC C \\
\hline & Mutado & CAC CTT GCT AAA GAAATT CTT GCT AA \\
\hline & Normal & GAT CAC TCC ACT GTT CAT AGG GAT CCAA \\
\hline \multirow[t]{2}{*}{ p.Asn1303Lys } & Común & СТС АAT TTC TTT ATT CTAAAG ACA TTG G \\
\hline & Mutado & GAT CAC TCC ACT GTT CAT AGG GAT CCA AC \\
\hline AAT1 & Control interno & TGT CCA CGT GAG CCT TGC TCG AGG CCT G \\
\hline AAT2 & Control interno & GAG ACT TGG TAT TTT GTT CAA TCA TTAAG \\
\hline AAT3 & Control interno & ССС АCС TTC ССС ТСТ СТC CAG GCAAAT G \\
\hline AAT4 & Control interno & GGG CCT CAG TCC CAA CAT GGC TAA GAG G \\
\hline
\end{tabular}

Fuente: Referencias bibliográficas ${ }^{(11,12)}$

Los productos de amplificación se separaron por electroforesis horizontal en gel de agarosa al $2 \%$ en una cámara electroforética (Cleaver scientific, Reino Unido) y se observaron en un transiluminador (Vilber lourmat, Francia). Los alelos normales y mutados fueron evidenciados por el número de pares de base (pb) y se compararon con controles positivos, obtenidos de Francia (p.Phe508del, p.Gly551Asp, p.Gly542*, p.Asn1303Lys); y controles internos AAT ALFA correspondientes al gen antitripsina ${ }^{(13)}$, estos permitieron validar el diagnóstico. 
Los tamaños de los amplicones obtenidos fueron estimados gracias al uso de marcador de peso molecular (MPM) de 100 pb obtenidos de Promega.

\section{ANÁLISIS ESTADÍSTICO}

Los datos obtenidos se analizaron utilizando estadística de tipo descriptiva. Los datos cualitativos fueron expresados como frecuencia y porcentaje.

\section{ASPECTOS ÉTICOS}

El presente estudio fue aprobado por los comités de ética del HNERM y el INSN. Se obtuvo el consentimiento informado escrito de cada padre o apoderado de los niños considerados en este estudio.

\section{RESULTADOS}

La búsqueda de casos para incluirlos en nuestro estudio nos permitió identificar al total de pacientes con FQ con atención médica en el Perú en el momento del estudio, los cuales fueron 49 pacientes. Algunos de estos ya tenían el diagnóstico genético que había sido realizado en laboratorios de fuera del país (Estados Unidos, Chile y Francia).

El presente estudio incluyó 36 pacientes peruanos con $F Q$. El rango de edad de los pacientes estuvo comprendido entre el 1 año 8 meses y los 19 años de edad. La edad promedio fue de 8 años 6 meses. Diecinueve casos $(52,8 \%)$ fueron varones. La procedencia de los pacientes correspondió a la ciudad de Lima con 26 casos (72,2\%) seguida de Arequipa con $2(5,6 \%)$, y otras ocho ciudades (Amazonas, Junín, Ancash, Cajamarca, Ica, Tacna, La Libertad y Loreto) que contribuyeron con un caso cada una $(2,8 \%)$.

Quince de los 36 pacientes (41.6\%) fueron positivos para al menos una de las diez mutaciones evaluadas. Asimismo, se detectaron tres (p.Phe508del, p.Gly542*, p.Arg1162*) de las diez mutaciones más frecuentes evaluadas. De los 72 alelos analizados, 22 fueron positivos $(30,6 \%)$. La mutación p.Phe508del fue la más frecuente y se encontró en 16 de un total de 72 alelos $(22,2 \%)$, la mutación p.Gly542* en cinco alelos $(6,9 \%)$, y la mutación p.Arg1162* en un alelo $(1,4 \%)$ (Tabla 2$)$.

Se identificaron mutaciones en ambos alelos en siete de 36 pacientes $(19,5 \%)$ y al menos una mutación en ocho de $36(22,2 \%)$ pacientes. No se encontraron mutaciones en 21 de 36 pacientes.
Tabla 2. Frecuencia alélica relativa de las mutaciones encontradas en los pacientes peruanos con FQ

\begin{tabular}{lcc}
\hline Mutaciones & $\begin{array}{c}\text { Alelos mutados } \\
\mathbf{n = 7 2}\end{array}$ & $\begin{array}{c}\text { Frecuencia alélica } \\
\mathbf{( \% )}\end{array}$ \\
\hline p.Phe508del & 16 & 22,2 \\
p.Gly542* & 5 & 6,9 \\
\hline p.Arg1162* & 1 & 1,4 \\
\hline ND & 50 & 69,4 \\
\hline
\end{tabular}

ND: no determinado; FQ: fibrosis quística

En el caso de frecuencia genotípica, el genotipo más frecuente fue el p.Phe508del/ND que estuvo presente en cinco de los 36 pacientes (13,9\%), seguido del genotipo p.Phe508del/p.Phe508del con cuatro pacientes $(11,1 \%)$ y p.Gly $542 * / N D$ con 3 pacientes $(8,33 \%$ ) (Tabla 3$)$.

\section{DISCUSIÓN}

A pesar de que los pacientes eran de diez ciudades del Perú, la mayoría de estos provenían de la ciudad de Lima, lo que podría explicarse porque en esta ciudad se encuentran la mayoría de instituciones que tratan la $\mathrm{FQ}$ y por tanto las que con más frecuencia detectarían la enfermedad.

Dentro de las diez mutaciones estudiadas, nuestro estudio solo encontró tres (p.Phe508del, p.Gly542* y p.Arg1162*). Estos resultados contrastan con lo que se esperaba encontrar al considerar las mutaciones más frecuentes de la región de acuerdo a un estudio publicado previamente ${ }^{(7)}$; y nos indicaría que la población de nuestro país podría presentar mutaciones diferentes, quizá producto del mestizaje u otro factor no considerado.

Tabla 3. Frecuencia genotípica relativa de las mutaciones encontradas en pacientes peruanos con fibrosis quística

\begin{tabular}{lccc}
\hline $\begin{array}{l}\text { Genotipo } \\
\text { alelo 1/alelo 2 }\end{array}$ & $\begin{array}{c}\text { Pacientes } \\
\text { con FQ }\end{array}$ & & $\begin{array}{c}\text { Frecuencia } \\
\text { genotípica }\end{array}$ \\
\cline { 2 - 2 } & $\mathbf{n = 3 6}$ & & $\mathbf{( \% )}$ \\
\hline p.Phe508del/p.Phe508del & 4 & & 11,1 \\
\hline p.Phe508del/p.Gly542* & 2 & 5,6 \\
p.Phe508del/p.Arg1162* & 1 & 2,8 \\
p.Phe508del/ND & 5 & 13,9 \\
p.Gly542*/ND & 3 & 8,3 \\
\hline ND/ND & 21 & 58,3 \\
\hline
\end{tabular}

ND: No determinado; FQ: fibrosis quística 
La mutación p.Phe508del, la más representativa a nivel mundial ${ }^{(2)}$, fue también la más común en nuestro estudio $(22.2 \%)$; sin embargo, al compararla con otros países, la frecuencia fue inferior al promedio latinoamericano $(46,7 \%)$ o a países como Argentina (59,2\%), Uruguay $(56,6 \%)$, Brasil $(43,1 \%)$, Chile $(39,3 \%)$, Cuba $(34,0 \%)$ y Ecuador $(31,4 \%)$, aunque fue ligeramente superior a Costa Rica $(22,9 \%)^{(7)}$.

La mutación p.Gly542* fue la segunda mutación más frecuente. Esta mutación se encuentra en el exón 11 del gen CFTR y pertenece a la clase de mutaciones I. Es la segunda más difundida en el mundo, se encuentra con una prevalencia más o menos constante y presenta una baja varianza entre poblaciones ${ }^{(14)}$. Su presencia en la población peruana se explicaría por la fuerte inmigración española que recibieron los países latinoamericanos. En nuestro estudio, esta mutación tuvo una frecuencia $(6,9 \%)$ más alta en comparación con Argentina (4,9\%) o Ecuador $(2 \%)$, similar a Brasil $(5,3 \%)$ y Chile $(5,6 \%)$, e inferior respecto a Uruguay $(7,9 \%)$ o Costa Rica, país que presenta una frecuencia de $25 \%{ }^{(7)}$.

La mutación p.Arg1162* fue la tercera mutación hallada en nuestro estudio (un alelo; 1,4\%) y su frecuencia fue mayor si se compara con países de Latinoamérica $(0,01 \%)$; sin embargo, esta prevalencia podría ser sobreestimada debido al tamaño relativamente reducido de la población estudiada, además que esta mutación solo fue encontrada en un paciente heterocigoto (Tabla 3). La combinación de un alelo mutado de tipo p.Arg1162* con otro de tipo p.Phe508del conduce a varios síntomas severos que incluyen la afección hepática; p.Arg1162* es una mutación sin sentido, muy común en el noreste de Italia ${ }^{(15)}$.

No pudimos detectar las mutaciones causantes de FQ en el $58,3 \%$ de los pacientes analizados. Esto se debió a que solo evaluamos las diez mutaciones más comunes en Latinoamérica y usamos un kit casero de ARMS-PCR. Es posible que los pacientes peruanos sean portadores de varias mutaciones y tengan una heterogeneidad genética alta tal como se ha mostrado en otros países latinoamericanos ${ }^{(16)}$.

Como dato adicional, se identificaron a once pacientes que ya tenían diagnóstico genético y cuyos resultados fueron colectados mediante encuestas directas realizadas por la asociación FIQUI Perú (9). De esta población todos los pacientes mostraron ser heterocigotos compuestos, siendo la mutación p.Phe508del la más frecuente (31,8\%; 7/22). Asimismo, en el mismo grupo se identificaron mutaciones raras como p.Arg1162*, p.GIn220*, p.Gly551Asp, p.Phe57Leu, c. 1116+2T>C, c. $2657+5 G>A$, p.Arg334Trp, p.Lys684Serfs*38, p.Arg347Pro y p.Lys1177Serfs*15, presentes como un alelo único. La mayor frecuencia del alelo p.Phe508del en comparación a nuestro estudio probablemente ocurra debido al pequeño tamaño de pacientes incluidos en este subgrupo y porque tres de estos pacientes eran hermanos, por lo tanto tenían las mismas mutaciones. Esta información no publicada en otra serie se suma a nuestros resultados, y ambos resaltan la heterogeneidad racial del país y confirmarían la hipótesis de que existen mutaciones poco comunes en nuestra población.

La identificación de las mutaciones presentes en los alelos del CFTR es de suma importancia para los pacientes, no solamente porque son confirmativas de la enfermedad sino también porque diferentes moléculas farmacéuticas han demostrado poder corregir completamente o atenuar los signos clínicos de la enfermedad, pero de forma específica a ciertas mutaciones ${ }^{(17,18)}$. Estos moduladores del CFTR incluyen potenciadores como el Ivacaftor VX-770 (nombre comercial: Kalydeco®) para pacientes con la mutación G551D, que incrementa el tráfico del cloruro por el canal del CFTR (mutaciones de clase III) ${ }^{(19)}$; correctores como el Lumacaftor VX-809 para pacientes con mutación p.Phe508del en forma homocigota, que mejora el plegamiento y el tráfico de la proteína CFTR anormal (mutaciones clase II) y que actualmente es comercializado como Orkambi ${ }^{\circledR}$ (lumacaftor + ivacaftor) ${ }^{(20)}$; y otras moléculas como el Ataluren, el cual está siendo evaluado en ensayos clínicos (nivel III), que corregiría las mutaciones que generan la aparición de un codón stop prematuro como la p.Arg1162* (mutaciones de clase I) ${ }^{(21,22)}$.

Este estudio permitió resaltar que las frecuencias de las mutaciones evaluadas fueron relativamente bajas $y$ que probablemente fueron adquiridas de descendientes europeos. Se requiere de otros estudios que permitan evaluar una mayor cantidad de mutaciones usando kits de ARMS-PCR que son comercialmente disponibles en otros países. No obstante, si analizamos las bajas coberturas que estos kits presentan para la población sudamericana, existe una clara necesidad de ampliar herramientas de secuenciamiento de ADN de próxima generación (en inglés: Next Generation Sequencing) (23) para aumentar las tasas de detección. Por otra parte, las herramientas de secuenciación directa del CFTR y la búsqueda de reordenamientos genómicos permitirán detectar posibles mutaciones prevalentes a nivel regional y mutaciones raras (privados) que no se encuentran con las pruebas de mutaciones específicas propuestas por los kits de ARMS-PCR ${ }^{(24,25)}$.

Nuestro estudio servirá como base para el establecimiento de futuras iniciativas de prevención que permitan el tamizaje de las mutaciones más frecuentes en la población general con el fin de identificar a 
eventuales portadores (hermanos, primos, etc.), futuros esposos, o permitir la fertilización in vitro con selección de embriones sanos en el caso de parejas portadoras.

En conjunto, la implementación en el país de herramientas de diagnóstico molecular permitiría la confirmación del diagnóstico, garantizaría un asesoramiento genético adecuado y lograría que los pacientes portadores de ciertas mutaciones puedan acceder a tratamientos curativos o paliativos que mejoren su calidad de vida ${ }^{(26)}$. Para luchar contra la FQ de forma más eficaz y mejorar la esperanza de vida de los pacientes peruanos, el país debería implementar una estrategia de diagnóstico sistemático con pruebas de tamizaje neonatal por IRT y establecer laboratorios de diagnóstico confirmativo por pruebas de sudor y equipos de diagnóstico molecular capaces de realizar pruebas genéticas de escaneo del gen CFTR.

Contribuciones de autoría: RAO, AP; JR, HA, MD, CN, NP, $\mathrm{BD}$ contribuyeron con la concepción del artículo, redacción del artículo y obtención del financiamiento. RAO, AP; JR, HA, MD $\mathrm{CN}$, NP contribuyeron con la recolección de los datos. RAO y $\mathrm{BD}$ realizaron el análisis de los datos. Todos aprobaron la versión final del manuscrito.

Fuentes de financiamiento: este trabajo fue financiado por los recursos del canon y sobrecanon entregados a la Universidad Nacional de Tumbes.

Conflicto de intereses: los autores declaran no tener ningún conflicto de interés.

\section{REFERENCIAS BIBLIOGRÁFICAS}

1. Kamath KS, Kumar SS, Kaur J, Venkatakrishnan V, Paulsen IT, Nevalainen $\mathrm{H}$, et al. Proteomics of hosts and pathogens in cystic fibrosis. Proteomics Clin Appl. 2015;9(1-2):134-46. doi: 10.1002/ prca.201400122

2. Fajac I, De Boeck K. New horizons for cystic fibrosis treatment. Pharmacol Ther. 2017;170:205-211. doi: 10.1016/j.pharmthera.2016.11.009

3. Sánchez K, Arcia O, MatuteX, Mindiola L, Chaustre I, Takiff H. Frequency of common CFTR gene mutations in Venezuelan patients with cystic fibrosis. Invest Clin. 2014; 55(1):44-54.

4. Rodas C, Gelvez N, Keyeux G. Mitochondrial DNA studies show asymmetrical Amerindian admixture in Afro-Colombian and Mestizo populations. Hum Biol. 2003;75(1):13-30.

5. Mateu E, Calafell F, Ramos MD, Casals T, Bertranpetit J. Can a place of origin of the main cystic fibrosis mutations be identified? Am J Hum Genet. 2002;70(1):257-64. doi: $10.1086 / 338243$

6. Silva Filho LV, Castaños C, Ruíz $\mathrm{HH}$. Cystic fibrosis in Latin AmericaImproving the awareness. J Cyst Fibros. 2016; 15(6):791-793. doi: 10.1016/j. jcf.2016.05.007

7. Pérez MM, Luna MC, Pivetta $\mathrm{OH}$, Keyeux G. CFTR gene analysis in Latin American CF patients: heterogeneous origin and distribution of mutations across the continent. J Cyst Fibros. 2007;6(3):194-208. doi: 10.1016/j. jcf.2006.07.004
8. Ashlock MA, Olson ER. Therapeutics development for cystic fibrosis: a successful model for a multisystem genetic disease. Annu Rev Med. 2011;62:107-25. doi: 10.1146/annurevmed-061509-131034

9. FIQUI Perú [Internet]. Lima: Asociación Peruana de Fibrosis Quística; c2016 [citado el 02 nov 2016]. Disponible en http://www.fiqui.org.pe/

10. Iranpur V, Esmailizadeh AK. Rapid Extraction of High Quality from Whole Blood. 2010 [Internet]. Irán: Shahrekord University; 2010 [citado el 02 nov 2016] Disponible en: http://www. protocol-online.org/prot/Protocols/ Rapid-Extraction-of-High-QualityDNA-from-Whole-Blood-Stored-at-4C-for-Long-Period-4175.html

11. Ferrie RM, Schwarz MJ, Robertson NH, Vaudin S, Super M, Malone G, Little S. Development, multiplexing, and application of ARMS tests for common mutations in the CFTR gene. Am J Hum Genet. 1992;51(2): 251-62.

12. Perone C, Medeiros GS, Del Castillo DM, De Aguiar MJ, Januário JN. Frequency of 8 CFTR gene mutations in cystic fibrosis patients in Minas Gerais, Brazil, diagnosed by neonatal screening. Braz J Med Biol Res. 2010;43(2):134-8.

13. Newton CR, Graham A, Heptinstall LE, Powell SJ, Summers C, Kalsheker $\mathrm{N}$, et al. Analysis of any point mutation in DNA. The amplification refractory mutation system (ARMS). Nucleic Acids Res. 1989;17(7): 2503-16.

14. Salazar S. Detection of mutations in the CFTR gene in the population of northeastern Mexico [tesis para obtener el grado de magister]. Monterrey: Universidad Autónoma de Nuevo León; 2003.

15. Shahin WA, Mehaney DA, ElFalaki MM. Mutation spectrum of Egyptian children with cystic fibrosis. Springerplus. 2016;5(1):686. doi: 10.1186/s40064-016-2338-7

16. Sánchez $\mathrm{K}$, de Mendonca E, Matute $\mathrm{X}$, Chaustre I, Villalón M, Takiff $\mathrm{H}$. Analysis of the CFTR gene in Venezuelan cystic fibrosis patients, identification of six novel cystic fibrosiscausing genetic variants. Appl Clin Genet. 2016;9:33-8. doi: 10.2147/ TACG.S78241

17. Amin R, Ratjen F. Emerging drugs for cystic fibrosis. Expert Opin Emerg Drugs. 2014;19(1):143-55. doi: $10.1517 / 14728214.2014 .882316$

18. Stewart C, Pepper MS. Cystic fibrosis on the African continent. Genet Med. 2016;18(7):653-62. doi: 10.1038/ $\operatorname{gim} .2015 .157$

19. Kapoor H, Koolwal A, Singh A. Ivacaftor: a novel mutation modulating drug. J Clin Diagn Res. 2014;8(11):SE01-5. doi: 10.7860/ JCDR/2014/6486.5158

20. Mayer M. Lumacaftor-ivacaftor (Orkambi) for cystic fibrosis: behind the 'breakthrough'. Evid Based Med. 2016;21(3): 83-6. doi: doi: 10.1136/ ebmed-2015-110325

21. Ryan NJ. Ataluren: first global approval. Drugs. 2014;74(14):1709-14. doi: $10.1007 / \mathrm{s} 40265-014-0287-4$ 
22. Bell SC, De Boeck K, Amaral MD. New pharmacological approaches for cystic fibrosis: promises, progress, pitfalls. Pharmacol Ther. 2015;145:19-34. doi: 10.1016/j.pharmthera.2014.06.005

23. Behjati S, Tarpey PS. What is next generation sequencing? Arch Dis Child Educ Pract Ed. 2013;98(6):236-8. doi: 10.1136/archdischild-2013-304340

24. Trujillano D, Ramos MD, González J, Tornador C, Sotillo F, Escaramis G, et al. Next generation diagnostics of cystic fibrosis and CFTR-related disorders by targeted multiplex high-coverage resequencing of CFTR. J Med Genet. 2013;50(7):455-62. doi: 10.1136/ jmedgenet-2013-101602

25. Straniero L, Soldà G, Costantino L, Seia M, Melotti P, Colombo C, Asselta R, Duga S. Whole-gene CFTR sequencing combined with digital RT-PCR improves genetic diagnosis of cystic fibrosis. J Hum Genet. 2016;61(12):977-84. doi: 10.1038/ jhg.2016.101

26. Lay-Son G, Puga A, Astudillo P, Repetto GM. Cystic fibrosis in Chilean patients: Analysis of 36 common
CFTR gene mutations. J Cyst Fibros. 2011;10(1):66-70. doi: 10.1016/j. jcf.2010.10.002

Correspondencia: Ruth Aquino Ordinola Dirección: Laboratorio de Biotecnología Molecular, Universidad Nacional de Tumbes. Av. Universitaria s/n, Pampa Grande. Tumbes, Perú.

Teléfono: (+51) 948359340

Correo electrónico: ruthaquinord@gmail.com

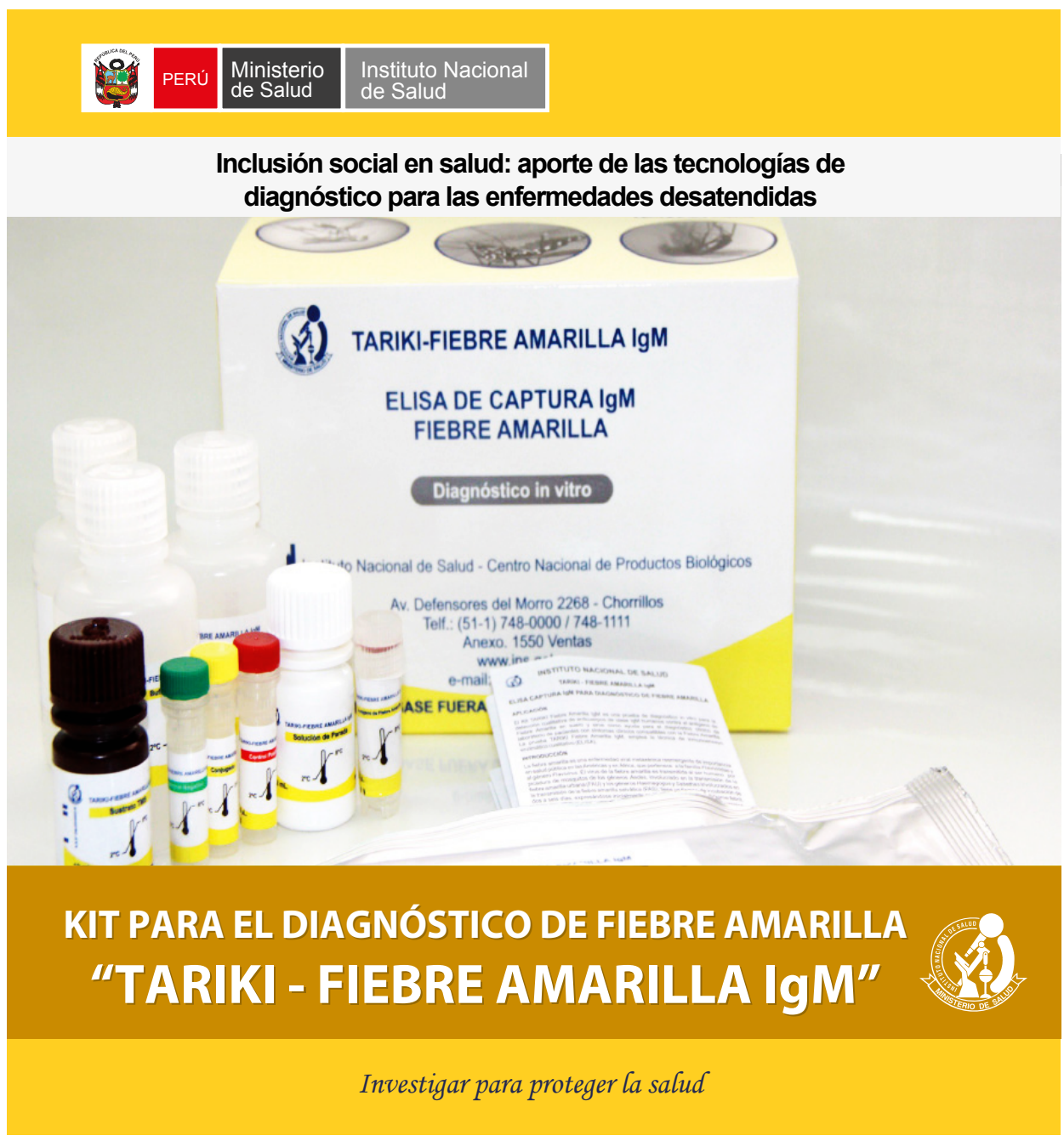

